Communications in Physics, Vol. 18, No. 1 (2007), pp. 1-8

\title{
HIGH TEMPERATURE SYMMETRY NON-RESTORATION AND INVERSE SYMMETRY BREAKING IN THE $Z_{2} \times Z_{2}$ MODEL
}

\author{
TRAN HUU PHAT \\ Vietnam Atomic Energy Commission, 59 Ly Thuong Kiet, Hanoi, Vietnam \\ LE VIET HOA \\ Hanoi National University of Education, 136 Xuan Thuy, Cau Giay, Hanoi, Vietnam \\ NGUYEN TUAN ANH \\ Institute for Nuclear Science and Technique, 5T-160 Hoang Quoc Viet, Hanoi \\ NGUYEN VAN LONG \\ Gialai Teacher College, 126 Le Thanh Ton, Pleiku, Gialai, Vietnam
}

\begin{abstract}
The patterns of high temperature symmetry non-restoration (SNR) and inverse symmetry braking (ISB) in the $Z_{2} \times Z_{2}$ model are investigated in detail for a specified parameters.
\end{abstract}

\section{INTRODUCTION}

At present it is well known that all physical systems can be classified into several categories:

a): The first one corresponds to those, in which the symmetry broken at $T=0$ is restored at high temperature [1-3]. In addition, there is another alternative phenomenon, the behavior of which associates with more broken symmetry as temperature is increased. This is the so-called inverse symmetry breaking (ISB). Here high temperature means that $T / M>>1$ for mass scale $\mathrm{M}$ of the system in question.

b): The second category deals with those cases which exhibit symmetry non-restoration (SNR) at high temperature. This phenomenon emerges in a lot of systems and materials [4]. In the context of quantum field theory, the high temperature SNR has been considered in [5-9] and recently developed in many papers in connection with various important cosmological applications [10-22].

In this respect, there remains growing interest on studying in [23], basing on the CJT effective action at finite temperature [24], we considered the $Z_{2} \times Z_{2}$ model, which was used in $[10,17]$ for the domain wall problem and in other Refs. $[25,26]$. This paper concerns a detailed investigation of phase transitions, which correspond to high temperature SNR/ISB of the $Z_{2} \times Z_{2}$ model for a specified set of the model parameters. In Section II, the main results of [23] are resumed. Section III is devoted for phase transition study. The conclusion and discussion are given in Section IV. 


\section{CONDITIONS FOR SNR/ISB}

Let us start from the system described by the simple Lagrangian

$$
£=\frac{1}{2}\left(\partial_{\mu} \phi\right)^{2}+\frac{\mu_{1}^{2}}{2} \phi^{2}+\frac{\lambda_{1}}{4 !} \phi^{4}+\frac{1}{2}\left(\partial_{\mu} \psi\right)^{2}+\frac{\mu_{2}^{2}}{2} \psi^{2}+\frac{\lambda_{2}}{4 !} \psi^{4}+\frac{\lambda}{4} \phi^{2} \psi^{2}+\Delta £ .
$$

The counter-terms are chosen as

$$
\Delta L=\frac{\delta \mu_{1}^{2}}{2} \phi^{2}+\frac{\delta \lambda_{1}}{4 !} \phi^{4}+\frac{\delta \mu_{2}^{2}}{2} \psi^{2}+\frac{\delta \lambda_{2}}{4 !} \psi^{4}+\frac{\delta \lambda}{4} \phi^{2} \psi^{2} .
$$

The boundedness of the potential appearing in (1) requires

$$
\lambda_{1}>0, \quad \lambda_{2}>0 \text { and } \lambda_{1} \lambda_{2}>9 \lambda^{2} .
$$

Shifting $\{\phi, \psi\} \rightarrow\left\{\phi+\phi_{0}, \psi+\psi_{0}\right\}$ leads to the interaction Lagrangian

$$
\begin{aligned}
£_{i n t} & =\frac{\lambda_{1}+\delta \lambda_{1}}{24} \phi^{4}+\frac{\lambda_{1}+\delta \lambda_{1}}{6} \phi_{0} \phi^{3}+\frac{\lambda_{2}+\delta \lambda_{2}}{24} \psi^{4}+\frac{\lambda_{2}+\delta \lambda_{2}}{6} \psi_{0} \psi^{3}+\frac{\lambda+\delta \lambda}{4} \phi^{2} \psi^{2} \\
& +\frac{\lambda+\delta \lambda}{2} \phi_{0} \phi \psi^{2}+\frac{\lambda+\delta \lambda}{2} \psi_{0} \psi \phi^{2}
\end{aligned}
$$

and the tree-level propagators

$$
\begin{aligned}
& D_{0}^{-1}\left(k ; \phi_{0}, \psi_{0}\right)=k^{2}+\mu_{1}^{2}+\delta \mu_{1}^{2}+\frac{\lambda_{1}+\delta \lambda_{1}}{2} \phi_{0}^{2}+\frac{\lambda+\delta \lambda}{2} \psi_{0}^{2}, \\
& G_{0}^{-1}\left(k ; \phi_{0}, \psi_{0}\right)=k^{2}+\mu_{2}^{2}+\delta \mu_{2}^{2}+\frac{\lambda_{2}+\delta \lambda_{2}}{2} \psi_{0}^{2}+\frac{\lambda+\delta \lambda}{2} \phi_{0}^{2} .
\end{aligned}
$$

Next the expressions for the renormalized CJT effective potential $V_{\beta}^{C J T}\left[\phi_{0}, \psi_{0}, D, G\right]$ and the gap equations at finite temperature are derived

$$
\begin{aligned}
V_{\beta}\left[\phi_{0}, \psi_{0}\right]= & \frac{\mu_{1 R}^{2}}{2} \phi_{0}^{2}+\frac{\lambda_{1 R}}{24} \phi_{0}^{4}+\frac{\mu_{2 R}^{2}}{2} \psi_{0}^{2}+\frac{\lambda_{2 R}}{24} \psi_{0}^{4}+\frac{\lambda_{R}}{4} \phi_{0}^{2} \psi_{0}^{2}+Q_{f}\left(M_{1 R}\right)+Q_{f}\left(M_{2 R}\right) \\
& -\frac{\lambda_{1 R}}{8}\left[P_{f}\left(M_{1 R}\right)\right]^{2}-\frac{\lambda_{2 R}}{8}\left[P_{f}\left(M_{2 R}\right)\right]^{2}-\frac{\lambda_{R}}{4} P_{f}\left(M_{1 R}\right) P_{f}\left(M_{2 R}\right), \\
& {\left[\mu_{1 R}^{2}+\frac{\lambda_{1 R}}{6} \phi_{0}^{2}+\frac{\lambda_{R}}{2} \psi_{0}^{2}+\frac{\lambda_{1 R}}{2} P_{f}\left(M_{1 R}\right)+\frac{\lambda_{R}}{2} P_{f}\left(M_{2 R}\right)\right] \phi_{0}=0 } \\
& {\left[\mu_{2 R}^{2}+\frac{\lambda_{2 R}}{6} \psi_{0}^{2}+\frac{\lambda_{R}}{2} \phi_{0}^{2}+\frac{\lambda_{2 R}}{2} P_{f}\left(M_{2 R}\right)+\frac{\lambda_{R}}{2} P_{f}\left(M_{1 R}\right)\right] \psi_{0}=0 . }
\end{aligned}
$$

and

$$
\begin{aligned}
& M_{1 R}^{2}=\mu_{1 R}^{2}+\frac{\lambda_{1 R}}{2}\left[\phi_{0}^{2}+P_{f}\left(M_{1 R}\right)\right]+\frac{\lambda_{R}}{2}\left[\psi_{0}^{2}+P_{f}\left(M_{2 R}\right)\right] \\
& M_{2 R}^{2}=\mu_{2 R}^{2}+\frac{\lambda_{2 R}}{2}\left[\psi_{0}^{2}+P_{f}\left(M_{2 R}\right)\right]+\frac{\lambda_{R}}{2}\left[\phi_{0}^{2}+P_{f}\left(M_{1 R}\right)\right] .
\end{aligned}
$$

where

$$
\begin{aligned}
& P_{f}(M)=\frac{M^{2}}{16 \pi^{2}} \ln \frac{M^{2}}{\mu^{2}}-\int \frac{d^{3} k}{(2 \pi)^{3}}\left[E(\vec{k})\left(1-e^{\frac{E(\vec{k})}{T}}\right)\right]^{-1} \\
& Q_{f}(M)=\frac{M^{4}}{64 \pi^{2}}\left(\ln \frac{M^{2}}{\mu^{2}}-\frac{1}{2}\right)+T \int \frac{d^{3} k}{(2 \pi)^{3}} \ln \left(1-e^{-\frac{E(\vec{k})}{T}}\right)
\end{aligned}
$$


Considering high temperature SNR/ISB let us assume that $\mu_{1}^{2}<0$ and $\mu_{2}^{2}>0$. As a consequence,

$$
\phi_{0} \neq 0 \text { and } \psi_{0}=0,
$$

which means that at $T=0$ symmetry of the system is spontaneously broken in $\phi$ sector and unbroken in $\psi$ sector.

It is easily obtained from (5) that the parameters are constrained by

$$
\begin{aligned}
& \lambda_{1}>0, \lambda_{2}>0, \mu_{1}^{2}<0, \mu_{2}^{2}>0, \\
& \lambda_{1} \lambda_{2}>9 \lambda^{2}, \lambda<0,|\lambda|>\lambda_{1}, \lambda_{2},
\end{aligned}
$$

for the present model, in which both SNR/ISB simultaneously take place at high temperatures in corresponding sector.

It was proved [23] that the constraints (6) for there being SNR/ISB is very stable in a large temperature interval due to the $\mathrm{T}$ logarithmic dependence of coupling constants.

\section{PHASE TRANSITION PATTERNS FOR SPECIFIED VALUES OF PARAMETERS}

In order to gain an insight into the model it is very interesting to consider the phase transitions for specified values of the model parameters. As is easily seen, there is no value of $\lambda$ which fulfils both conditions

$$
\lambda_{1} \lambda_{2}>9 \lambda^{2}, \quad|\lambda|>\lambda_{1}, \lambda_{2} .
$$

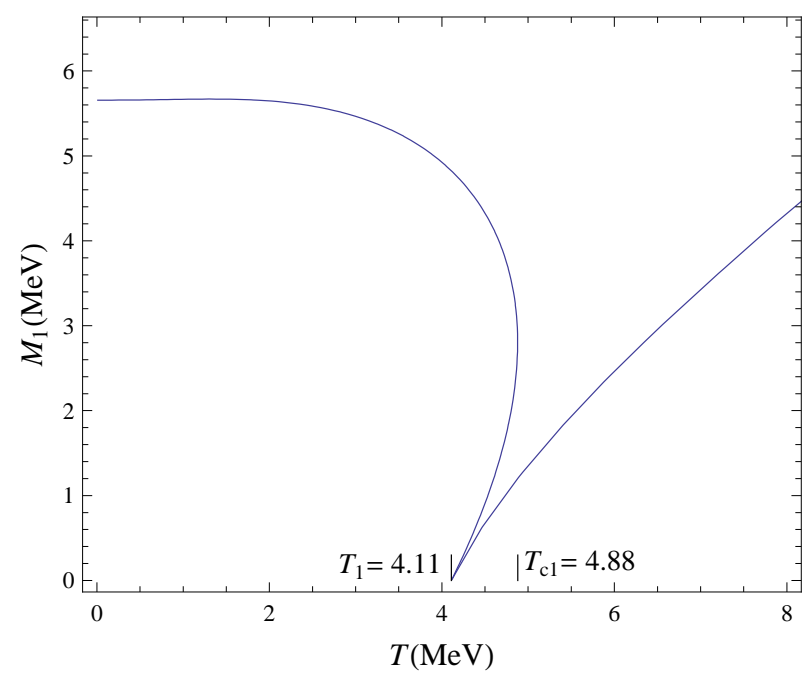

Fig. 1. The $T$ dependence of $M_{1}$, corresponding to the region that the broken symmetry in $\phi$-sector is restored (see Fig. 3 ). The phase transition happens in the interval $\left[T_{1}, T_{c 1}\right]$.

In this respect, let us proceed to the phase transitions study for the case, in which broken symmetry gets restored in $\phi$ sector and ISB takes place in $\psi$ sector. Accordingly, 


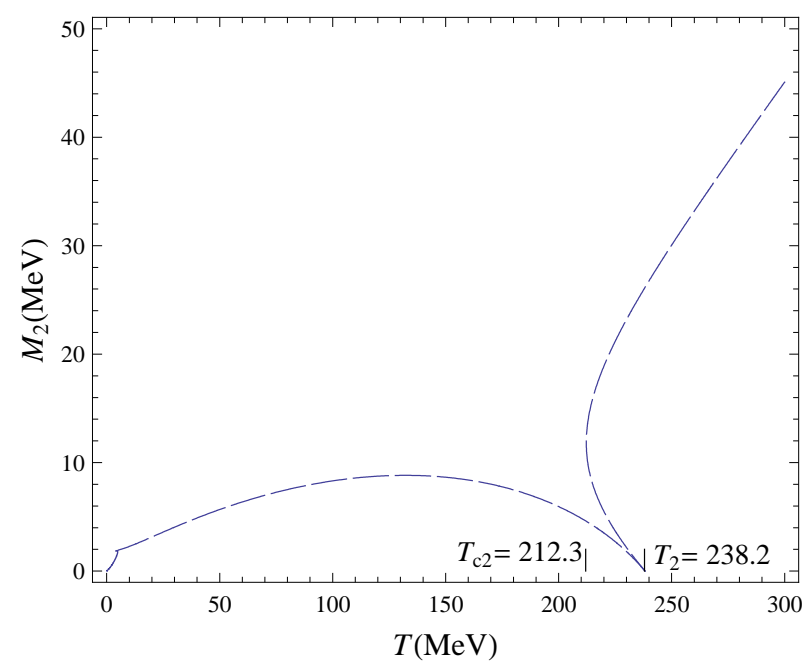

Fig. 2. The $T$ dependence of $M_{2}$, corresponding to the region that the symmetry in $\psi$-sector is broken (see Fig. 5). The phase transition happens in the interval $\left[T_{c 2}, T_{2}\right]$.

the parameters are constrained as follows.

$$
\begin{aligned}
& \lambda_{1}>0, \lambda_{2}>0, \mu_{1}^{2}<0, \mu_{2}^{2}>0, \\
& \lambda<0, \quad \lambda_{1}>|\lambda|>\lambda_{2}, \quad \lambda_{1} \lambda_{2}>9 \lambda^{2} .
\end{aligned}
$$

For illustration let us choose at random some specified values for $\mu_{1}^{2}, \mu_{2}^{2}, \lambda_{1}, \lambda_{2}$ and $\lambda$, which obey the above mentioned inequalities:

$$
\begin{aligned}
& \mu_{1}^{2}=-(4 \mathrm{MeV})^{2}, \quad \mu_{2}^{2}=(2 \mathrm{MeV})^{2}, \\
& \lambda_{1}=24, \quad \lambda_{2}=1,8 \quad \text { and } \quad \lambda=-2 .
\end{aligned}
$$

They are the inputs for numerical computations. We first remark that, in addition to the model parameters, the renormalization introduced another parameter $\mu$, which is the renormalization scale. Then we must determine a suitable value $\mu_{0}^{2}$ of $\mu^{2}$, which is defined as the real root of the following equation

$$
\left.\phi_{0}\left(\mu^{2}, 0\right)\right|_{\mu^{2}=\mu_{0}^{2}}=2 \mathrm{MeV},
$$

where $\phi_{0}\left(\mu^{2}, 0\right)$ is a solution of the system of Eqs. (4) and (5) at $T=0$. The numerical computation gives $\mu_{0}=5.657 \mathrm{MeV}$.

In $\phi$-sector, eliminating $\phi_{0}$ from (4) and (5) leads to

$$
\begin{aligned}
& M_{1}^{2}(T)=-2 \mu_{1}^{2}-\lambda_{1} P_{f}\left(M_{1}\right)-\lambda P_{f}\left(M_{2}\right) \\
& M_{2}^{2}(T)=\mu_{2}^{2}+\frac{3 \lambda}{\lambda_{1}} \mu_{1}^{2}-\lambda P_{f}\left(M_{1}\right)+\left(\frac{\lambda_{2}}{2}-\frac{6 \lambda^{2}}{4 \lambda_{1}}\right) P_{f}\left(M_{2}\right) .
\end{aligned}
$$




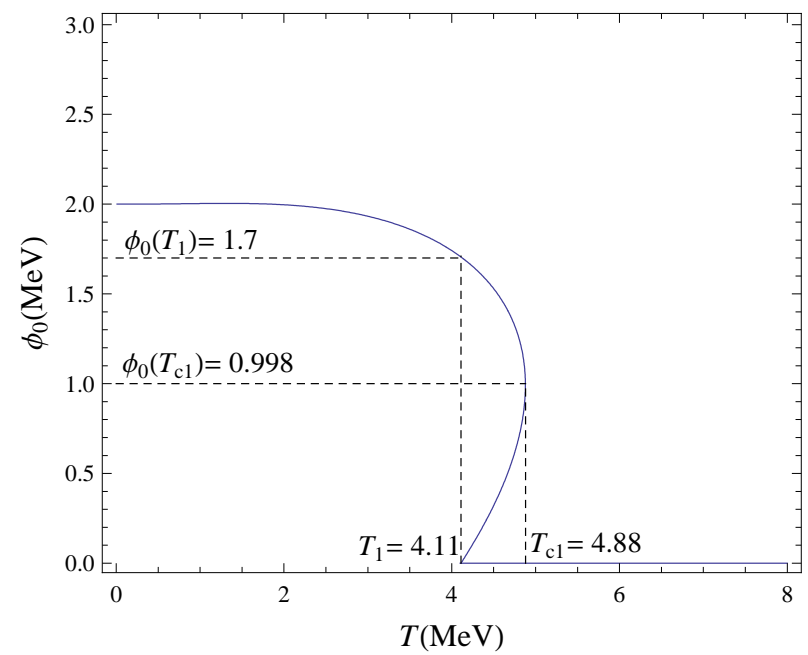

Fig. 3. The $T$ evolution of the order parameter $\phi$, in which the broken symmetry in $\phi$-sector is restored. The phase transition happens in the interval $\left[T_{1}, T_{c 1}\right]$. At $T_{1}$, the value $\phi_{0}=0$ is in maximum of $V\left(\phi_{0}, T\right)$, while the value $\phi_{0}=1.7 \mathrm{MeV}$ is in minimum. In the interval $T_{1}<T<T_{c 1}$, the value $\phi_{0}=0$ is in minimum at $V\left(\phi_{0}, T\right)=0$, value $\phi_{02}$ is in maximum, and $\phi_{01}$ in minimum. At $T_{c 1}$, there is an inflexion point of $V\left(\phi_{0}, T\right)$ at $\phi_{0}=0.988 \mathrm{MeV}$. (see Fig. 4).

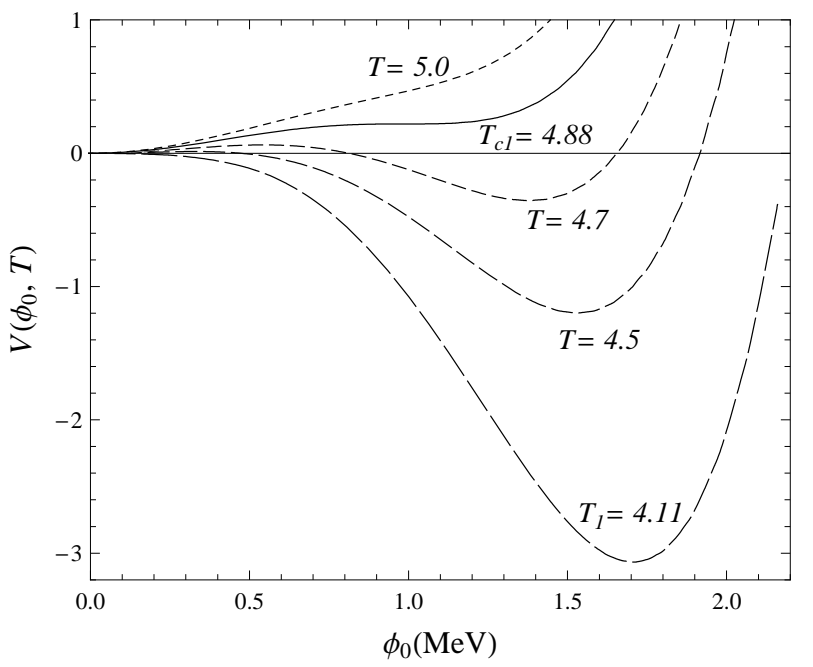

Fig. 4. The evolution of the $V\left(\phi_{0}, T\right)$ as a function of the order parameter $\phi_{0}$ for several temperature steps: $T=4.11,4.5,4.7,4.878,5 . \mathrm{MeV}$ from bottom to top. At $T_{1}$, the value $\phi_{0}=0$ is in maximum of $V\left(\phi_{0}, T\right)$, while the value $\phi_{0}=1.7 \mathrm{MeV}$ is in minimum. In the interval $T_{1}<T<T_{c 1}$, the value $\phi_{0}=0$ is in minimum at $V\left(\phi_{0}, T\right)=0$, value $\phi_{02}$ is in maximum, and $\phi_{01}$ in minimum (see Fig. 4). At $T_{c 1}$, there is an inflexion point of $V\left(\phi_{0}, T\right)$ at $\phi_{0}=0.988 \mathrm{MeV}$. 
for $\phi_{0} \neq 0$, and

$$
\begin{aligned}
& M_{1}^{2}(T)=\mu_{1}^{2}+\frac{\lambda_{1}}{2} P_{f}\left(M_{1}\right)+\frac{\lambda}{2} P_{f}\left(M_{2}\right), \\
& M_{2}^{2}(T)=\mu_{2}^{2}+\frac{\lambda_{2}}{2} P_{f}\left(M_{2}\right)+\frac{\lambda}{2} P_{f}\left(M_{1}\right) .
\end{aligned}
$$

for $\phi_{0}=0$.

Inserting $\mu^{2}=\mu_{0}^{2}$ into (8) and then solving numerically this system of equations we obtain the solutions $M_{1}$ in Fig. 1, and similar to $\psi$-sector we have $M_{2}$ presented in Fig. 2 .

The $\mathrm{T}$ dependence of the order parameter $\phi_{0}$ is given in Fig. 3. It is observed in these figures that for $0<T<T_{1} \approx 4.18 \mathrm{MeV}$ a first order phase transition persists. When $T=T_{1}$ a second order phase transition emerges and in the interval $T_{1} \leq T \leq T_{c 1}$ both phase transitions coexist up to $T_{c 1} \approx 4.878 \mathrm{MeV}$, at which

$$
\left.\frac{d \phi_{0}(T)}{d T}\right|_{T=T_{c 1}}=\infty
$$

$T_{c 1}$ is exactly the critical temperature, where the system transform from first order phase transition to second order one. This phenomenon is highlighted by means of the numerical computation performed for $V_{\beta}\left[\phi_{0}, \psi_{0}=0\right]$, as function of $\phi_{0}$ at several values of $T$. It is easily proved that the curve, corresponding to $T=T_{c 1}=4.878 \mathrm{MeV}$ in Fig. 4, has an inflexion point at $\phi_{0}\left(T_{c 1}\right)=0.998 \mathrm{MeV}$ and $V\left[\phi_{0}\left(T_{c 1}\right)\right]=0.227 \mathrm{MeV}$. The broken symmetry is then restored at $T_{c 1}$.

In order to consider the high temperature ISB in $\psi$ sector the $\mathrm{T}$ dependence of $\psi_{0}(T)$ for large $T$ are plotted in Fig. 5.

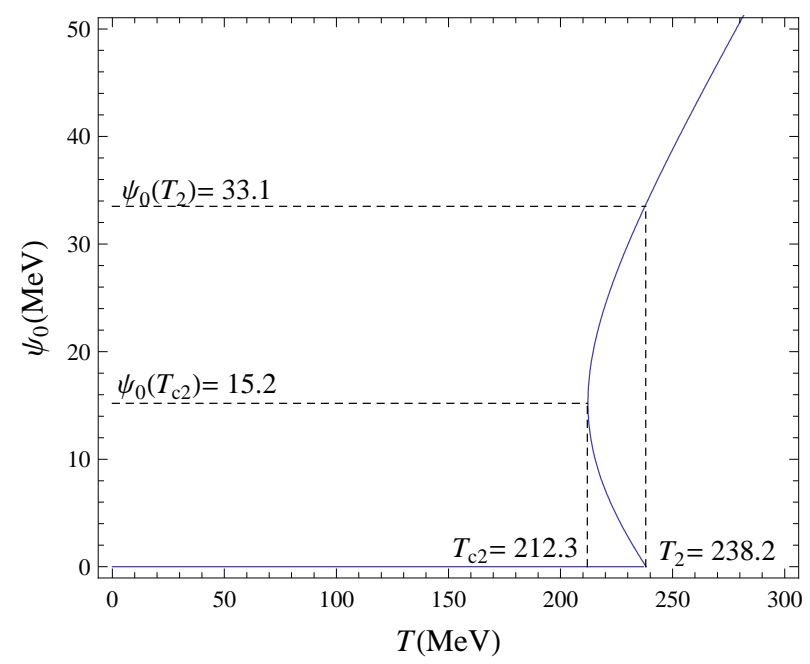

Fig. 5. The $T$ evolution of the order parameter $\psi$, in which the symmetry in $\psi$-sector is broken. The phase transition happens in the interval $\left[T_{c 2}, T_{2}\right]$. At $T_{2}$, the value $\psi_{0}=0$ is in maximum of $V\left(\psi_{0}, T\right)$, while the value $\psi_{0}=33.1 \mathrm{MeV}$ is in minimum. In the interval $T_{c 2}<T<T_{2}$, the value $\psi_{0}=0$ is in minimum at $V\left(\psi_{0}, T\right)=0$, value $\psi_{02}$ is in maximum, and $\psi_{01}$ in minimum. At $T_{c 2}$, there is an inflexion point of $V\left(\psi_{0}, T\right)$ at $\psi_{0}=15.2 \mathrm{MeV}$. (see Fig. 6). 


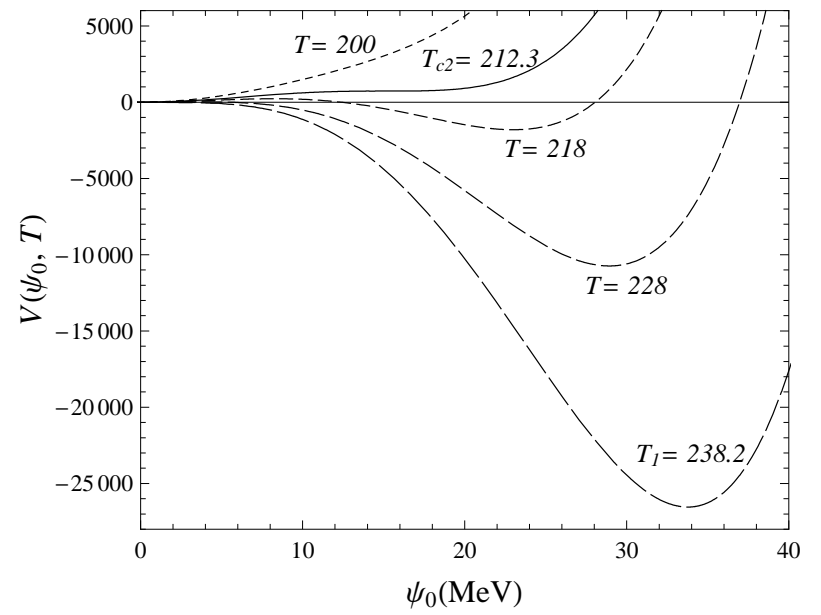

Fig. 6. The evolution of the $V\left(\psi_{0}, T\right)$ as a function of the order parameter $\psi_{0}$ for several temperature steps: $T=200,212.253,218,228,238.232 \mathrm{MeV}$ from top to bottom. At $T_{2}$, the value $\psi_{0}=0$ is in maximum of $V\left(\psi_{0}, T\right)$, while the value $\psi_{0}=33.1 \mathrm{MeV}$ is in minimum. In the interval $T_{c 2}<T<T_{2}$, the value $\psi_{0}=0$ is in minimum at $V\left(\psi_{0}, T\right)=0$, value $\psi_{02}$ is in maximum, and $\psi_{01}$ in minimum (see Fig. 5). At $T_{c 2}$, there is an inflexion point of $V\left(\psi_{0}, T\right)$ at $\psi_{0}=15.2 \mathrm{MeV}$.

It is evident that the symmetry is broken for $T=T_{c 2}=212.253 \mathrm{MeV}$, at which

$$
\left.\frac{d \psi_{0}(T)}{d T}\right|_{T=T_{c 2}}=\infty
$$

$T_{c 2}$ is the critical temperature when the system exhibits simultaneously first and second order phase transition. It is the temperature for ISB to take place in $\psi$ sector. The evolution of $V_{\beta}\left[\phi_{0}=0, \psi_{0}\right]$ against $\psi_{0}$ for different temperatures is shown in Fig. 6 . It is properly asserted that the inflection point of the curve $T=T_{c 2}=212.253 \mathrm{MeV}$ possesses coordinates $\psi_{0}\left(T_{c 2}\right)=15.230 \mathrm{MeV}$ and $V_{\beta}\left[\psi_{0}\left(T_{c 2}\right)\right]=789.02 \mathrm{MeV}$.

\section{CONCLUSION AND DISCUSSION}

In this paper the phase transitions were considered for $Z_{2} \times Z_{2}$ model by means of the finite temperature CJT effective action. We investigated in detail phase transitions for a set of parameter chosen at random. The numerical solutions for the gap equations and the shape of effective potential, as function of order parameters at different temperatures, exhibit the coexistence of first and second order phase transitions for SNR in $\phi$ sector and ISB in $\psi$ sector. Although the model studied earlier is too simple, but all those we observed in the preceding section are extremely interesting and their main feature does not depend on the chosen set of parameters, provided the latter obeys (7), of course. The generalization to $O(M) \times O(N)$-model is straightforward and produces similar results. Our present study, in some sense, could be considered to be complementary to those obtained in $[6,13,14,25,26]$.

\section{ACKNOWLEDGMENTS}

This paper was financially supported by Vietnam National Foundation for Scientific Research. 


\section{REFERENCES}

[1] D. Kirznhits and A. Linde, Phys. Lett. B42 (1972) 471.

[2] L. Dolan and R. Jackiw, Phys. Rev. D9 (1974) 3320.

[3] S. Weinberg, Phys. Rev. D9 (1974) 3357.

[4] N. Schupper and N.M. Shnerb, Phys. Rev. E72 (2005) 046107.

[5] G. Bimonte and G. Lozano, Phys. Lett. B366 (1996) 248; Nucl. Phys. B460 (1996) 155.

[6] T.G. Roos, Phys. Rev. D54 (1996) 2944.

[7] M. Pietroni, N. Rius and N. Tetrdis, Phys. Lett. B397 (1997) 119.

[8] G. Bimonte, D. Iniguez, A. Taracon and C.L. Ullod, Nucl. Phys. B559 (1999) 108.

[9] G. Amelino-Camelia, Nucl. Phys. B476 (1996) 255.

[10] R. N. Mohapatra and G. Senjanovic, Phys. Rev. Lett. 42 (1979) 1651; Phys. Rev. D20 (1979) 3390.

[11] G. Dvali, A. Melfo and G. Senjanovic, Phys. Rev. Lett. 75 (1995) 4559.

[12] P. Langacker and S.Y. Pi, Phys. Rev. Lett. 45 (1980) 1.

[13] M. B. Pinto and R. O. Ramos, Phys. Rev. D61 (2000) 125016.

[14] M. B. Pinto, R. O. Ramos and J.E. Parreira, Phys. Rev. D71 (2005) 123519.

[15] J. P. Preskill, Phys. Rev. Lett. 43 (1979) 1365.

[16] Ya. B. Zeldovich, I. Yu. Kobzarev and L. B. Okun, JETP 40 (1974) 1.

[17] G. Dvali and G. Senjanovic, Phys. Rev. Lett. 74 (1995) 5178.

[18] A. Masiero and G. Senjanovic, Phys. Lett. B108 (1982) 191.

[19] R. N. Mohapatra and G. Senjanovic, Phys. Rev. D21 (1980) 3470.

[20] H. Georgi, Hadronic J., 1 (1978) 155.

[21] M. A. B. Beg and H. S. Tsao, Phys. Rev. Lett. 41 (1978) 278.

[22] R. N. Mohapatra and G. Senjanovic, Phys. Lett. 79B (1978) 28.

[23] Tran Huu Phat, Le Viet Hoa, Nguyen Tuan Anh and Nguyen Van Long, Phys. Rev. D 76 (2007) 125027.

[24] G. Amelino-Camelia and S. Y. Pi, Phys. Rev. D47 (1993) 2356.

[25] S. Bornholdt, N. Tetradis and C. Wetterich, Phys. Rev. D53 (1996) 4552.

[26] M. Pietroni, N. Rius and N. Tetradis, Phys. Lett. B397 (1997) 119. 
